\title{
A riqueza de espécies arbóreas na floresta atlântica de encosta no estado de São Paulo (Brasil)
}

\author{
MARCELO TABARELLI ${ }^{1}$ e WALDIR MANTOVANI ${ }^{2}$
}

(recebido em 24/04/97; aceito em 24/02/99)

\begin{abstract}
Woody species richness in the Brazilian atlantic forest, state of São Paulo (Brazil)). The atlantic forest in São Paulo state, has been described in the scientific literature as having high floristic diversity. Such statement is based on data and conclusions of local floristic and phytosociological studies. However, the studies supporting this idea seldom give a clear definition of the plant group analysed, the scale of the study, nor, most significantly, the forests used for comparisons. Here we compare woody species richness data $(\geq 2.5 \mathrm{~cm} \mathrm{dbh}$ ) from the atlantic forest in São Paulo to similar data from other neotropical forests. Thereafter, we discuss the major relationships between historical and ecological processes and woody species richness in neotropical forests. Neither data on woody species richness nor characteristics of latitude, altitude, rainfall and soil in the atlantic region is able to support the idea of high woody species richness in the São Paulo atlantic forest. Studies using comparable data sets are required in order to characterise woody species richness in all types of the atlantic forest, as well as to suggest the mechanisms responsible for plant richness in different scales.
\end{abstract}

RESUMO - (A riqueza de espécies arbóreas na floresta atlântica de encosta no estado de São Paulo (Brasil)). Extensa literatura referese à floresta atlântica de encosta no estado de São Paulo como possuidora de elevada diversidade florística. Esta afirmação está baseada em dados e conclusões de estudos florísticos e fitossociológicos locais. A maioria destes trabalhos não define com clareza o que considera diversidade, de que grupos estão tratando, a escala de abordagem e, principalmente, quais as florestas usadas para comparação. Neste estudo, são comparados dados sobre riqueza de plantas arbóreas ( $\geq 2,5 \mathrm{~cm}$ dap) na floresta atlântica de encosta em São Paulo com os de outras florestas neotropicais. Posteriormente, são discutidas as principais relações conhecidas entre a riqueza de plantas lenhosas nas florestas neotropicais e fatores históricos e ecológicos. Nem os dados sobre riqueza nem as características de latitude, altitude, precipitação e solo na região da encosta atlântica suportam a hipótese de riqueza elevada nesta floresta em São Paulo. Estudos realizados a partir de base de dados comparáveis são necessários para caracterizar a riqueza de espécies lenhosas em todos os tipos de floresta, bem como para sugerir hipóteses sobre os processos responsáveis pelos padrões observados em diferentes escalas.

Key words - Atlantic rain forest, species richness, woody plants, floristic composition

\section{Introdução}

A área costeira do Brasil, considerando-se as formações sobre as restingas e a floresta atlântica, representa uma região com índice elevado de endemismos (Thomaz et al. 1998), onde ocorrem, por exemplo, 39 espécies endêmicas de Chrysobalanaceae (Prance 1987). Das 127 espécies lenhosas descritas para região costeira na Flora Neotropica, 68 (53,5\%) são endêmicas (Mori et al. 1981). De acordo com diversos autores (Mori et al. 1983, Peixoto \& Gentry 1990, Joly et al. 1991, Barros et al. 1991), além do elevado grau de endemismo observado em alguns grupos vegetais, a floresta atlântica apresenta elevada riqueza de espécies e diversidade florística (sensu Begon et al. 1996), que, em alguns locais, é superior às observadas em trechos de floresta amazônica (Silva \& Leitão Filho 1982, Martins 1989, Brown Jr. \& Brown 1992).

1. Departamento de Botânica, Universidade Federal de Pernambuco, Rua Moraes Rego s/n, 50670-901 Recife, PE, Brasil.

2. Departamento de Ecologia Geral, Universidade de São Paulo, Caixa Postal 11461, 05408-400 São Paulo, SP, Brasil.
A floresta atlântica não é uma formação homogênea. Conforme Joly et al. (1991), a floresta atlântica (Sul/Sudeste) é composta por três formações distintas: as matas das planícies litorâneas, as matas de encosta e as matas de altitude. Esta classificação pode ser estendida para todos os domínios da floresta atlântica na costa brasileira. Nas regiões Sul e Sudeste, com exceção do estado do Espírito Santo, predomina a floresta de encosta e, na região Nordeste, a floresta de terras baixas (Rizzini 1979). Do ponto de vista fitogeográfico, esta floresta é composta por dois blocos distintos, um formado pela região Nordeste e outro, pela região Sudeste/Sul. O estado do Espírito Santo abriga uma flora intermediária entre os dois (Siqueira 1994).

Conforme Mantovani (1993), o conjunto de estudos fitossociológicos e florísticos efetuados na floresta atlântica de encosta no estado de São Paulo (i.e, floresta ombrófila densa) tem sugerido elevada riqueza e diversidade de espécies arbóreo-arbustivas. A riqueza de hábitats, ocupados por diferentes fisionomias florestais, tem reforçado a idéia de riqueza e diversidade elevadas (Leitão Filho 1994). Grande 
parte da literatura (por exemplo, Silva \& Leitão Filho 1982, Martins 1989, Mantovani 1990, Joly et al. 1991, Leitão Filho 1994, Siqueira 1994) refere-se à floresta atlântica de encosta como possuidora de elevada diversidade florística. A maioria dos trabalhos não define com clareza o que considera diversidade, de que grupos estão tratando, a escala de abordagem e, principalmente, qual é o controle adotado para as comparações. A análise de alguns estudos (por exemplo, Mantovani et al. 1990, Leitão Filho 1993, Mantovani 1993, Sanches 1994) sugere que a floresta atlântica de encosta no estado de São Paulo apresenta riqueza de espécies arbóreas reduzida nos níveis local e regional, comparativamente às outras florestas neotropicais conhecidas.

O objetivo deste trabalho foi analisar as informações existentes sobre a riqueza de espécies arbóreas ( $\geq 2,5 \mathrm{~cm}$ dap) na floresta atlântica de encosta no estado de São Paulo. Usando abordagens simples, como a comparação entre o número de espécies amostradas por unidade de área, são apresentadas evidências de que esta floresta apresenta riqueza de plantas arbóreas, nos níveis local e regional, inferior a observada em várias outras florestas neotropicais.

\section{Material e métodos}

Floresta atlântica - Considerou-se como floresta atlântica somente a floresta ombrófila densa, estabelecida na costa atlântica brasileira. Neste estudo, o termo floresta de encosta refere-se àquelas estabelecidas sobre relevo montanhoso, as quais ocorrem preferencialmente dentro dos limites da floresta baixo-montana e montana, ou seja entre 50 e $1500 \mathrm{~m}$ de altitude (Veloso \& GóesFilho 1982).

Escala de análise - A riqueza de espécies arbóreas ( $\geq 2,5 \mathrm{~cm}$ dap) foi analisada nos níveis local e regional. A riqueza no nível local corresponde à diversidade alfa (sensu Whittaker 1972), sendo neste estudo, aquela observada em levantamentos através dos métodos de parcelas (áreas amostrais entre 0,1 e 1,0 ha) ou de quadrantes (Valencia et al. 1994). A riqueza no nível regional corresponde ao total de espécies amostradas em regiões definidas no texto. O termo arbóreo neste trabalho inclui plantas lenhosas comumente classificadas como grandes arbustos, arvoretas e árvores.

Seleção de levantamentos - Para a caracterização da riqueza de espécies arbóreas $(\geq 2,5 \mathrm{~cm}$ dap) na floresta atlântica de encosta, no estado de São Paulo, foram selecionados levantamentos efetuados em florestas maduras (sensu Saldarriaga et al. 1988), estabelecidas dentro dos domínios da floresta ombrófila densa, distribuídos entre $23^{\circ}$ e $25^{\circ}$ de latitude sul. Neste trabalho, foi incluído um estudo inédito realizado pelos autores no Núcleo Santa Virgínia, em Ubatuba, SP. Este estudo teve como base o estabelecimento de uma parcela de
$100 \times 100 \mathrm{~m}$ (1 ha) em trecho de floresta madura $(900 \mathrm{~m}$ de altitude), onde todos os indivíduos com diâmetro a altura do peito (dap) igual ou superior a $10 \mathrm{~cm}$ foram amostrados. O material botânico coletado foi identificado no Instituto de Botânica, SP, e está depositado na coleção de referência do Laboratório de Fitossociologia e Dinâmica de Populações da Universidade de São Paulo, SP. Maiores informações sobre as características da vegetação no Núcleo Santa Virgínia podem ser obtidas em Tabarelli (1997).

Como controle, foram selecionados estudos realizados na floresta atlântica de terras baixas nas regiões Sudeste (ES) e Nordeste do Brasil, floresta amazônica e outras florestas na América do Sul. Todos os trechos estudados pertencem a florestas úmidas $(>1400 \mathrm{~mm} / \mathrm{ano})$, de terras baixas $(<500 \mathrm{~m}$ de altitude) e estabelecidas sobre solos férteis ou com fertilidade intermediária. Optou-se somente por estudos recentes, para restringir a influência de problemas taxonômicos e de subestimativa da riqueza de espécies, comuns nos estudos feitos há mais tempo, onde as espécies eram identificadas por nomes vulgares. Para a análise da riqueza local, as florestas foram comparadas entre si em termos de número de espécies amostradas por unidade de área ou por número total de indivíduos amostrados. A riqueza regional foi analisada comparando-se o número total de espécies observadas em sete levantamentos na floresta atlântica de encosta em São Paulo com a riqueza observada em levantamentos locais em outras florestas neotropicais.

\section{Resultados}

Riqueza local - A tabela 1 apresenta o número de espécies de árvores $(\geq 10 \mathrm{~cm}$ dap) observado em várias florestas neotropicais. Estas florestas podem ser classificadas arbitrariamente em três níveis de riqueza: 1) florestas com menos de 200 espécies/ha; 2) florestas que contêm entre 200 e 300 espécies/ha e 3) florestas com mais de 300 espécies/ha.

De acordo com os resultados apresentados na tabela 1, a floresta atlântica de encosta enquadra-se no menor nível de riqueza, conjuntamente com florestas do Xingu, na Amazônia brasileira e com um trecho de floresta amazônica na Bolívia. Outras comparações podem ser feitas, de forma direta, entre a riqueza observada em sítios de floresta na encosta atlântica e as de outras florestas neotropicais. Melo \& Mantovani (1994), na Ilha do Cardoso, SP, numa área amostral dez vezes maior, encontraram 72,6\% do número de espécies amostradas por Peixoto \& Gentry (1990), em Linhares, ES, utilizando critério de inclusão semelhante. Gentry \& Terborgh (1990), numa área amostral de 0,1 ha, em Cocha Cashu, no Peru, encontraram 78,3\% do número de espécies encontradas por Melo \& Mantovani (1994). Comparando-se estudos realizados através do método de quadrantes, Mori et al. (1983) amostraram 600 ár- 
Tabela 1. Riqueza de espécies arbóreas na floresta atlântica de encosta e em outras florestas neotropicais na América do Sul.

\begin{tabular}{|c|c|c|c|c|c|}
\hline Local & Método & $\begin{array}{l}\text { Área } \\
\text { (ha) }\end{array}$ & $\begin{array}{l}\text { Dap } \\
(\mathrm{cm})\end{array}$ & $\begin{array}{c}\mathrm{N}^{\circ} \\
\text { espécies }\end{array}$ & Fonte \\
\hline \multicolumn{6}{|l|}{ Floresta atlântica } \\
\hline Ubatuba & parcelas & 1,0 & $\geq 10$ & 136 & M.Tabarelli, dados não publicados \\
\hline Ilha Cardoso & parcelas & 1,0 & $>2,6$ & 157 & Melo \& Mantovani (1994) \\
\hline \multicolumn{6}{|l|}{ Outras florestas } \\
\hline Cuyabeno, Equador & parcelas & 1,0 & $\geq 10$ & 307 & Valencia et al. (1994) \\
\hline Yanamomo, Peru & parcelas & 1,0 & $\geq 10$ & 283 & Gentry (1988b) \\
\hline Mishana, Peru & parcelas & 1,0 & $\geq 10$ & 275 & Gentry (1988b) \\
\hline Juruá 4, Brasil & parcelas & 1,0 & $\geq 10$ & 271 & Silva et al. (1992) \\
\hline Juruá 3, Brasil & parcelas & 1,0 & $\geq 10$ & 260 & Silva et al. (1992) \\
\hline Juruá 2, Brasil & parcelas & 1,0 & $\geq 10$ & 224 & Silva et al. (1992) \\
\hline Juruá 1, Brasil & parcelas & 1,0 & $\geq 10$ & 213 & Silva et al. (1992) \\
\hline Belém, Brasil & parcelas & 1,0 & $\geq 10$ & 196 & Almeida et al. (1993) \\
\hline Belém, Brasil & parcelas & 1,0 & $\geq 10$ & 191 & Almeida et al. (1993) \\
\hline Cocha Cashu, Peru & parcelas & 1,0 & $\geq 10$ & 189 & Gentry (1988b) \\
\hline Belém, Brasil & parcelas & 1,0 & $\geq 10$ & 179 & Almeida et al. (1993) \\
\hline Manaus, Brasil & parcelas & 1,0 & $\geq 15$ & 179 & Prance et al. (1976) \\
\hline Cabeza Mono, Peru & parcelas & 1,0 & $\geq 10$ & 169 & Gentry (1988b) \\
\hline Tambopata 1, Peru & parcelas & 1,0 & $\geq 10$ & 168 & Gentry (1988b) \\
\hline Xingu 2, Brasil & parcelas & 1,0 & $\geq 10$ & 162 & Campbell et al. (1986) \\
\hline Tambopata 2, Peru & parcelas & 1,0 & $\geq 10$ & 155 & Gentry (1988b) \\
\hline Belém, Brasil & parcelas & 1,0 & $\geq 10$ & 147 & Almeida et al. (1993) \\
\hline Xingu 1, Brasil & parcelas & 1,0 & $\geq 10$ & 133 & Campbell et al. (1986) \\
\hline Xingu 3, Brasil & parcelas & 1,0 & $\geq 10$ & 118 & Campbell et al. (1986) \\
\hline Alto Ivon, Bolívia & parcelas & 1,0 & $\geq 10$ & 98 & Boom (1986) \\
\hline Cocha Cashu, Peru & parcelas & 0,1 & $\geq 2,5$ & 123 & Gentry \& Terborgh (1990) \\
\hline Linhares, Brasil & parcelas & 0,1 & $\geq 2,5$ & $216^{*}$ & Peixoto \& Gentry (1990) \\
\hline
\end{tabular}

* incluindo lianas

vores (dap $\geq 10 \mathrm{~cm}$ ) em um trecho de floresta atlântica de terras baixas na Bahia, onde encontraram 178 espécies. Silva \& Leitão Filho (1982) amostraram 640 árvores (dap $\geq 10$ ), em dois trechos de floresta atlântica de encosta em São Paulo, onde encontraram 104 espécies.

Riqueza regional - Na tabela 2 estão sete estudos realizados na floresta atlântica de encosta no estado de São Paulo. No total, foram amostradas 432 espécies arbóreas em uma área de aproximadamente 2,3 ha, mais a amostragem de 2640 indivíduos através do método de quadrantes. O conjunto destas espécies representa uma amostra da riqueza desta floresta no estado de São Paulo. A análise da tabela 3 indica que grande parte da riqueza de espécies da floresta de encosta deve-se a Melastomataceae, Rubiaceae, Myrsinaceae, Myrtaceae, Monimiaceae, Palmae e Flacourtiaceae.
Em 0,1 ha de floresta atlântica de terras baixas no Espírito Santo, Peixoto \& Gentry (1990) encontraram 216 espécies lenhosas $(\geq 2,5 \mathrm{~cm}$ dap) que equivalem a $52,4 \%$ do número de espécies amostradas nos sete levantamentos, em uma área 25 vezes maior na floresta atlântica de encosta (tabela 4). Em várias outras florestas onde a composição de espécies é conhecida, a riqueza encontrada em parcelas de 0,1 e 1 ha representa parte significativa da que foi observada no nível regional na floresta atlântica de encosta em São Paulo (tabela 4), o que sugere que esta floresta apresenta menor riqueza de espécies também no nível regional. Nas florestas citadas na tabela 4, destacam-se famílias como Sapotaceae, Burseraceae, Chrysobalanaceae, Moraceae, Lecythidaceae e Leguminosae. Este pequeno grupo de famílias parece responsável pelas diferenças de riqueza entre 
Tabela 2. Levantamentos florísticos realizados na floresta atlântica de encosta no estado de São Paulo*.

\begin{tabular}{lccccl}
\hline \multicolumn{1}{c}{ Local } & Método & Área & Dap $(\mathrm{cm})$ & $\begin{array}{c}\mathrm{N}^{\circ} \\
\text { espécies }\end{array}$ & \multicolumn{1}{c}{ Fonte } \\
\hline Juréia & parcelas & 0,5 ha & $>4,8$ & 107 & Melo, dados não publicados \\
Ilha Cardoso & parcelas & 1,0 ha & $>2,6$ & 157 & Melo \& Mantovani (1994) \\
Cubatão & parcelas & 0,4 ha & $>5,5$ & 126 & Leitão Filho (1993) \\
Picinguaba & parcelas & 0,4 ha & $>5,0$ & 96 & Sanches (1994) \\
Juréia & quadrantes & 200 pontos & $>9,5$ & 155 & Mantovani (1993) \\
Juréia & quadrantes & 200 pontos & $>1,59<9,5$ & 89 & Mantovani (1993) \\
Salesópolis & quadrantes & 100 pontos & $>4,8$ & 104 & Mantovani et al. (1990) \\
Ubatuba & quadrantes & 160 pontos & $\geq 10$ & 104 & Silva \& Leitão Filho (1982) \\
\hline
\end{tabular}

* Adaptado de Siqueira (1994)

a floresta atlântica de encosta e as demais florestas analisadas (tabelas 3 e 4).

\section{Discussão}

Os resultados apresentados neste estudo não suportam a hipótese de riqueza elevada de espécies arbóreas na floresta atlântica de encosta no estado de São Paulo, nas diferentes escalas com que a mesma foi analisada. Ao contrário, constituem-se em evidências de que a floresta atlântica de encosta apresenta menor riqueza comparativamente às outras florestas neotropicais conhecidas. Os resultados apresentados por Guedes-Bruni et al. (1997) ampliam estas evidências, pois os autores amostraram apenas 187 espécies em 1 ha de floresta atlântica de encosta em Macaé de Cima, RJ, apesar do critério de inclusão dos indivíduos ter sido $5 \mathrm{~cm}$ de dap.

Padrões de riqueza de espécies podem ser conseqüência de diversos processos históricos e ecológicos, que atuam ou atuaram em diferentes escalas de espaço e tempo (Schluter \& Ricklefs 1993). Entre os fatores históricos, destaca-se a localização dos centros de riqueza ou distribuição (sensu Gentry 1982). Com exceção de Myrtaceae, as famílias com maior riqueza de plantas lenhosas nas florestas neotropicais têm seus principais centros de riqueza fora da região costeira brasileira. Com maior riqueza de arbustos destacam-se Melastomataceae, Monimiaceae, Myrsinaceae, Piperaceae, Rubiaceae e Solanaceae, famílias com principal centro de riqueza no norte dos Andes (Gentry 1982). Entre as árvores destacam-se Leguminosae, Annonaceae, Chrysobalanaceae, Lecythidaceae, Sapotaceae,
Euphorbiaceae e Bignoniaceae (lianas), famílias que, no Novo Mundo, apresentam seu principal centro de riqueza na região Amazônica (Gentry 1982, 1988a).

A riqueza de plantas lenhosas nas florestas tropicais está relacionada a cinco gradientes principais: o latitudinal, o de precipitação, o edáfico, o altitudinal e o intercontinental (Gentry 1988a, Clinebell II et al. 1995). Nas florestas neotropicais, observa-se uma relação direta entre a precipitação, a fertilidade dos solos e a riqueza de espécies. Observa-se um decréscimo de riqueza com o aumento da latitude e altitude, mantidas as outras condições ecológicas e biogeográficas constantes (Gentry 1988a). Conforme este autor, observa-se uma tendência clara de decréscimo de riqueza com o aumento da altitude, principalmente de árvores. Famílias ricas em árvores, como Leguminosae, Sapotaceae, Myristicaceae, Meliaceae, Sapindaceae, Burseraceae, Chrysobalanaceae e, em árvores e lianas, como Bignoniaceae, são pobremente representadas nas florestas neotropicais montanas (i.e, $>600-800 \mathrm{~m}$ de altitude).

Em gradientes altitudinais nos Andes, a partir das florestas de terras baixas, Lauraceae substitui Leguminosae, sendo uma das famílias com maior riqueza de espécies em elevações intermediárias. Entre as famílias com maior riqueza nas médias elevações (800-1500 m) estão Melastomataceae, Myrtaceae, Rubiaceae, Euphorbiaceae, Moraceae e Guttiferae, com muitas espécies de arbustos e pequenas árvores (Gentry 1988a). Em altitudes elevadas (> $2000 \mathrm{~m}$ ) predominam Melastomataceae, Compositae, Rubiaceae, Aquifoliaceae, Myrtaceae e Theaceae. A riqueza observada em outros locais (por exemplo, Heaney \& Proctor 1990, Nadkarni et al. 1995) suporta a hi- 
Tabela 3. Principais famílias com pelo menos quatro espécies amostradas em sete levantamentos florísticos na floresta atlântica de encosta no estado de São Paulo.

\begin{tabular}{lc}
\hline \multicolumn{1}{c}{ Famílias } & $\mathrm{n}^{\text {o }}$ de espécies \\
\hline Myrtaceae & 79 \\
Leguminosae & 49 \\
Rubiaceae & 35 \\
Lauraceae & 27 \\
Melastomataceae & 17 \\
Euphorbiaceae & 16 \\
Sapotaceae & 13 \\
Annonaceae & 11 \\
Moraceae & 10 \\
Chrysobalanaceae & 10 \\
Bignoniaceae & 9 \\
Sapindaceae & 9 \\
Monimiaceae & 8 \\
Myrsinaceae & 8 \\
Palmae & 7 \\
Proteaceae & 7 \\
Meliaceae & 6 \\
Flacourtiaceae & 6 \\
Rutaceae & 6 \\
Aquifoliaceae & 5 \\
Vochysiaceae & 5 \\
Cecropiaceae & 5 \\
Clusiaceae & 532 \\
Piperaceae & 4 \\
Boraginaceae & \\
\hline \multicolumn{1}{c}{ Total de espécies amostradas } & \\
\hline
\end{tabular}

pótese de menor riqueza de árvores nas florestas neotropicais montanas.

Ao contrário das florestas montanas, a elevada riqueza de árvores parece ser o padrão para as florestas neotropicais de terras baixas na América do Sul. Estudos realizados em diferentes locais na floresta Amazônica encontraram entre 118 e 307 espécies de árvores ( $\geq 10 \mathrm{~cm}$ dap) por hectare. Bombacaceae, Burseraceae, Chrysobalanaceae, Lauraceae, Lecythidaceae, Leguminosae, Moraceae, Sapotaceae e Vochysiaceae são as famílias com maior riqueza de árvores naquelas florestas (Veloso 1946, Prance et al. 1976, Mori \& Boom 1983, Campbell et al. 1986, Gentry \& Terborgh 1990). As árvores ( $\geq 10 \mathrm{~cm}$ dap) constituem somente entre 15 e $22 \%$ das espécies nas florestas neotropicais onde há floras conhecidas (Gentry \& Dodson 1987). O restante são lianas, epífitas, ervas, arbustos e pequenas árvores que nunca atingem $10 \mathrm{~cm}$ de dap. Gentry \& Emmons (1987) constataram uma relação significativa entre fertilidade de solo, precipitação total, duração da estação seca e riqueza de espécies de sub-bosque (ervas, arbustos e arvoretas) e riqueza de epífitas (Gentry \& Dodson 1987, Gentry 1990).

Com o decréscimo da fertilidade e/ou precipitação, ou aumento da estação seca, observa-se redução na riqueza, primeiramente de ervas, seguido das epífitas e, por último, de arbustos e pequenas árvores.

Tabela 4. Famílias com maior riqueza em espécies arbóreas (S) em cinco trechos de florestas neotropicais¹.

\begin{tabular}{|c|c|c|c|c|c|c|c|c|c|}
\hline \multicolumn{2}{|c|}{ Cuyabeno-PE ${ }^{2}$} & \multicolumn{2}{|l|}{ Juruá-BR ${ }^{2}$} & \multicolumn{2}{|c|}{ Linhares-BR ${ }^{3}$} & \multicolumn{2}{|c|}{ Belém-BR ${ }^{2}$} & \multicolumn{2}{|c|}{ Manaus-BR ${ }^{4}$} \\
\hline Família & S & Família & S & Família & S & Família & S & Família & S \\
\hline Leguminosae & 33 & Leguminosae & 49 & Leguminosae & 32 & Leguminosae & 37 & Leguminosae & 20 \\
\hline Lauraceae & 29 & Sapotaceae & 23 & Myrtaceae & 31 & Sapotaceae & 25 & Lecythidaceae & 18 \\
\hline Sapotaceae & 25 & Chrysobalanaceae & 21 & Sapotaceae & 13 & Burseraceae & 13 & Moraceae & 13 \\
\hline Burseraceae & 20 & Vochysiaceae & 14 & Bignoniaceae & 11 & Chrysobalanaceae & 12 & Sapotaceae & 12 \\
\hline Moraceae & 21 & Burseraceae & 11 & Lauraceae & 9 & Lecythidaceae & 9 & Chrysobalanaceae & 10 \\
\hline Chrysobalanaceae & 14 & Euphorbiaceae & 10 & Hipocrateaceae & 8 & Moraceae & 9 & Burseraceae & 10 \\
\hline Euphorbiaceae & 13 & Lauraceae & 13 & Euphorbiaceae & 8 & Guttiferae & 7 & Euphorbiaceae & 10 \\
\hline Myrtaceae & 10 & Moraceae & 12 & Apocynaceae & 6 & Lauraceae & 7 & Lauraceae & 7 \\
\hline Cecropiaceae & 10 & Annonaceae & 9 & Rubiaceae & 5 & Annonaceae & 6 & Annonaceae & 7 \\
\hline Myristicaceae & 12 & Myristicaceae & 8 & Nyctaginaceae & 5 & Apocynaceae & 6 & Vochysiaceae & 7 \\
\hline Total de espécies & 307 & & 260 & & 216 & & 179 & & 165 \\
\hline
\end{tabular}

${ }^{1}$ Referências conforme tabela $1 ;{ }^{2} 1$ ha, dap $\geq 10 \mathrm{~cm} ;{ }^{3} 0,1$ ha, dap $\geq 2,5 \mathrm{~cm} ;{ }^{4} 1$ ha, dap $\geq 15 \mathrm{~cm}$ 
Além da riqueza, com o aumento nos níveis de precipitação, constata-se a elevação nos níveis de contribuição relativa de epífitas, ervas, lianas, arbustos e pequenas árvores na riqueza geral da floresta (Gentry 1990). As florestas com maiores riquezas de epífitas, ervas, lianas, arbustos e pequenas árvores recebem entre 2980 e $7470 \mathrm{~mm} /$ ano de precipitação, estão sob solos com níveis altos ou intermediários de fertilidade (1300-8000 ppm de N) e sem estação seca definida (Gentry \& Dodson 1987, Gentry \& Emmons 1987, Gentry 1990). Exemplos são La Selva na Costa Rica, Rio Palenque e Jauneche, no Equador, todas de terras baixas $(<220 \mathrm{~m}$ de altitude). Entre os fatores ecológicos associados a diferenças de riqueza, destaca-se, também, a variação latitudinal (Gentry 1988a, Huston 1994). Gentry (1988a) demostrou que acima de $20^{\circ}$ de latitude, ocorre decréscimo acentuado na riqueza de plantas lenhosas nas florestas tropicais.

A maior parte da floresta atlântica de encosta no estado de São Paulo e no sul do Brasil recebe precipitações inferiores a $3000 \mathrm{~mm} / \mathrm{ano}$, encontra-se sobre solos pobres ou com níveis intermediários de fertilidade (Mantovani 1993) e está estabelecida preferencialmente entre $300 \mathrm{~m}$ e $1200 \mathrm{~m}$ de altitude e acima de $20^{\circ}$ de latitude, características que estão relacionadas à redução da riqueza de plantas lenhosas em florestas neotropicais. Ou seja, tanto os dados sobre riqueza, como as características de localização, precipitação e solo na encosta atlântica não suportam a hipótese de riqueza elevada nesta floresta, apesar da mesma compor o centro de endemismo São Paulo-Rio de Janeiro (sensu Thomas et al. 1998).

As generalizações feitas sobre a riqueza e/ou diversidade da floresta atlântica brasileira refletem o fato de que, apesar der ser considerada uma das maiores prioridades mundiais para conservação (Biodiversity Support Program et al. 1995), pouca informação comparável está disponível sobre esta floresta. A importância biológica de alguns trechos da floresta atlântica parece estar relacionada aos níveis de endemismo e não à riqueza total de espécies em diferentes escalas. Estudos realizados a partir de base de dados comparáveis são necessários para caracterizar a riqueza de espécies em diferentes escalas e nos diferentes tipos desta floresta, bem como para sugerir hipóteses sobre os mecanismos responsáveis pelos padrões observados.

\section{Referências bibliográficas}

ALMEIDA, S.S., LISBOA, P.L. \& SILVA, A.S.L. 1993. Diversidade florística de uma comunidade arbórea na Estação Científica "Ferreira Penna, em Caxiuanã (Pará). Bol. Mus. Para. Emílio Goeldi 9:93-128.

BARROS, F., MELO, M.M.R.F., CHIEA, S.A.C., KIRIZAWA, M., WANDERLEY, M.G.L., \& JUNG-MENDAÇOLLI, S.L. 1991. Caracterização geral da vegetação e listagem das espécies ocorrentes. In Flora Fanerogâmica da Ilha do Cardoso (M.M.R.F. Melo et al., ed.). Instituto de Botânica, São Paulo. v1.

BEGON, M., HARPER, J.L. \& TOWNSEND, C.R. 1996. Ecology: individuals, populations and communities. Blackwell, Oxford.

BIODIVERSITY SUPPORT PROGRAM, CONSERVATION INTERNATIONAL, THE NATURE CONSERVANCY, WILDLIFE CONSERVATION SOCIETY, WORLD RESOURCES INSTITUTE \& WORLD WILDLIFE FUND. 1995. A regional analysis of geographic priorities for biodiversity conservation in Latin America and Caribbean. Biodiversity Support Program, Washington, DC.

BOOM, B.M. 1986. Forest inventory in Amazonian Bolivia. Biotropica 18:287-294.

BROWN JR., K.S. \& BROWN, G.G. 1992. Habitat alteration and species loss in Brazilian forests. In Tropical deforestation and species extinction (T.C. Whitmore \& J.A. Sayer, eds.). Chapman \& Hall, London, p.129-142.

CAMPBELL, D.G., DALY, D.G., PRANCE, G.T. \& MACIEL, U.N. 1986. Quantitative ecological inventory of terra firme and várzea tropical forest on the rio Xingu, Brazilian Amazon. Brittonia 38:369-393.

CLINEBELL II, R.R., PHILLIPS, O.L., GENTRY, A.H., STARK, N. \& ZUURING, H. 1995. Prediction of neotropical tree and liana species richness from soil and climatic data. Biol. Conserv. 4:56-90.

GENTRY, A.H. 1982. Neotropical floristic diversity: phytogeographical connections between Central and South America, pleistocene climatic fluctuations, or an accident of the andean orogeny? Ann. Missouri. Bot. Gard. 69:557-593.

GENTRY, A.H. 1988a. Changes in plant community diversity and floristic composition on environmental and geographical gradients. Ann. Missouri Bot. Gar. 75:1-34.

GENTRY, A.H. 1988b. Tree species richness of upper amazonian forests. Proc. Natl. Acad. Sci. 85:156-159.

GENTRY, A.H. 1990. Floristic similarities and differences between Southern Central America and upper and Central Amazonia. In Four neotropical rain forests (A.H. Gentry, ed.). Yale University Press, London, p.141-160.

GENTRY, A.H. \& DODSON, C. 1987. Contribuition of nontrees to species richness of a tropical rain forest. Biotropica 19:149-156.

GENTRY, A.H. \& EMMONS, L.H. 1987. Geographical variation in fertility, phenology, and composition of the understory of neotropical forests. Biotropica 19:216-227.

GENTRY, A.H. \& TERBORGH, J. 1990. Composition and dynamics of the Cocha Cashu "mature" foodplain forest. In Four neotropical rain forests (A.H. Gentry, ed.). Yale University Press, London, p.543-563.

GUEDES-BRUNI, R.R., PESSOA, S.V.A. \& KURTZ, B.C. 1997. Florística e estrutura do componente arbustivo-arbóreo de um trecho preservado de floresta montana na Reserva Ecológica de Macaé de Cima. In Serra de Macaé de Cima: diversidade florística e conservação em Mata Atlântica (H.C. Lima \& R.R. Guedes-Bruni, eds.). Jardim Botânico do Rio de Janeiro, Rio de Janeiro, p.127-146. 
HEANEY, A. \& PROCTOR, J. 1990. Preliminary studies on forest structure and floristic on Volcan Barva, Costa Rica. J. Trop. Ecol. 11:481-495.

HUSTON, A.H. 1994. Biological diversity, the coexistence of species on changing landscapes. Cambridge University Press, London.

JOLY, C.A., LEITÃO FILHO, H.F. \& SILVA, S.M. 1991. O patrimônio florístico - The floristic heritage. In Mata Attântica atlantic rain forest (G.I. Câmara, coord.). Ed. Index Ltda. e Fundação S.O.S. Mata Attântica, São Paulo.

LEITÃO FILHO, H.F. (org.). 1993. Ecologia da Mata Atlântica em Cubatão. Editora da UNESP e Editora da Universidade de Campinas, Campinas.

LEITÃO FILHO, H.F. 1994. Diversity of arboreal species in atlantic rain forest. An. Acad. Bras. Ci. 66 (supl. 1):91-96.

MANTOVANI, W. 1990. A dinâmica da floresta na encosta atlântica. Anais do II simpósio de ecossistemas da costa sul e sudeste brasileira: estrutura, função e manejo. ACIESP, São Paulo, v.1:304-313.

MANTOVANI, W. 1993. Estrutura e dinâmica da floresta atlântica na Juréia, Iguape-SP. Tese de livre-docência, Universidade de São Paulo, São Paulo.

MANTOVANI, W., RODRIGUES, R.R., ROSSI, L., ROMANIUC-NETO, S., CATHARINO, E.L.M. \& CORDEIRO, I 1990. A vegetação na Serra do Mar em Salesópolis. Anais do II simpósio de ecossistemas da costa sul e sudeste brasileira estrutura, função e manejo. ACIESP, São Paulo, v.2:348-384

MARTINS, R.F. 1989. Fitossociologia de florestas do Brasil: um histórico bibliográfico. Pesq. sér. Bot. 40:105-161.

MELO, M.R.F. \& MANTOVANI, W. 1994. Composição florística e estrutura fitossociológica da mata atlântica de encosta na Ilha do Cardoso (Cananéia, Brasil). Bol. Inst. Bot. 9:107-158

MORI, S.A., BOOM, B.M. \& PRANCE, G.T. 1981. Distrubuition patterns and conservation of eastern Brazilian coastal forest species. Brittonia 33:233-245.

MORI, S.A. \& BOOM, B.M. 1983. Southern bahian moist forests. Bot. Rev. 49:155-232.

MORI, S.A., BOOM, B.M., CARVALINO, A.M. \& SANTOS, T.S. 1983. Ecological importance of Myrtaceae in a eastern Brazilian wet forest. Biotropica 15:68-70.

NADKARNI, N.M., MATELSON, T.J. \& HARBER, W.A. 1995. Structural characteristics and floristic composition of a neotropical cloud forest, Monteverde, Costa Rica. J. Trop. Ecol. 11:481-495.

PEIXOTO, A.L. \& GENTRY, A. 1990. Diversidade e composição florística da mata de tabuleiro na Reserva Florestal de Linhares (Espírito Santo, Brasil). Revta brasil. Bot. 13:19-25.
PRANCE, G.T. 1987. Biogeography of neotropical plants. In Biogeography and quaternary history in tropical America (T.C Whitmore \& G.T. Prance, eds.). Claredon Press, Oxford, p.175-196.

PRANCE, G.T., RODRIGUES, W.A. \& DA SILVA, M.F. 1976. Inventário florestal de um hectare de mata de terra firme km 30 da estrada Manaus-Itacoatiara. Acta Amaz. 6:9-35.

RIZZINI, C.T. 1979. Tratado de fitogeografia do Brasil: aspectos sociológicos e florísticos. EDUSP e Editora HUCITEC, São Paulo.

SALDARRIAGA, J.G., WEST, D.C., THARP, M.L. \& UHL, C. 1988. Long-term chronosequence in the upper rio Negro of Colombia and Venezuela. J. Ecol. 76:938-958.

SANCHES, M. 1994. Florística e fitossociologia da vegetação arbórea nas margens do rio da Fazenda (P.E.S.M.), Ubatuba, São Paulo. Dissertação de mestrado, UNESP, Rio Claro.

SCHLUTER, D. \& RICKLEFS, R.E. 1993. Species diversity: an introduction to the problem. In Species diversity in ecological communities: historical and geographical perspectives (R.E. Ricklefs \& D. Schluter, eds.). University of Chicago Press, Chicago, p.1-10.

SILVA, A.F. \& LEITÃO FILHO, H.F. 1982. Composição florística e estrutura de um trecho da mata atlântica de encosta no município de Ubatuba (São Paulo, Brasil). Revta brasil. Bot. 5:43-52.

SILVA, A.S.L., LISBOA, P.B. \& MACIEL, U.B. 1992. Diversidade florística e estrutura em floresta densa da bacia do rio JuruáAM. Bol. Mus. Para. Emílio Goeldi 8:203-258.

SIQUEIRA, M.F. 1994. Análise florística e ordenação de espécies arbóreas da Mata Atlântica através de dados binários. Dissertação de Mestrado, Universidade Estadual de Campinas, Campinas.

TABARELLI, M. 1997. A regeneração da floresta Atlântica montana. Tese de doutorado, Universidade de São Paulo, São Paulo.

THOMAS, W.W., CARVALHO, A.M.A., GARRISON, J. \& ARBELAEZ, A.L. 1998. Plant endemism in two forests in southern Bahia, Brazil. Biod. Conser. 7:311-322.

VALENCIA, R., BALSLEV, H. \& PAZ Y MIÑO, G. 1994. High tree alpha-diversity in Amazonian Ecuador. Biodiver. Conser. 3:21-28.

VELOSO, H.P. 1946. A vegetação no município de Ilhéus, estado da Bahia. I. Estudo sinecológico das áreas de pesquisas sobre a febre amarela silvestre realizado pelo SEPFA. Mem. Inst. Oswal. Cruz 44:13-103.

VELOSO, H.P. \& GOES-FILHO, L. 1982. Classificação fisionômico-ecológica da vegetação neotropical. Bol. Téc. Proj. Radambrasil v.7.

WHITTAKER, R.H. 1972. Evolution and measurement of species diversity. Taxon 21:213-251. 